\title{
Monoclonal antibodies for the treatment of non- haematological tumours: update of an expanding scenario
}

Giuseppe Bronte, Giovanni Sortino, Francesco Passiglia, Sergio Rizzo, Francesca Lo Vullo, Antonio Galvano, Viviana Bazan, Christian Rolfo \& Antonio Russo MD PhD

To cite this article: Giuseppe Bronte, Giovanni Sortino, Francesco Passiglia, Sergio Rizzo, Francesca Lo Vullo, Antonio Galvano, Viviana Bazan, Christian Rolfo \& Antonio Russo MD PhD (2015) Monoclonal antibodies for the treatment of non-haematological tumours: update of an expanding scenario, Expert Opinion on Biological Therapy, 15:1, 45-59, DOI: 10.1517/14712598.2015.963052

To link to this article: http://dx.doi.org/10.1517/14712598.2015.963052

Published online: 24 Sep 2014.

Submit your article to this journal $匹$

Џ Article views: 173

Q View related articles ¿

View Crossmark data ¿ 


\section{EXPERT OPINION}

1. Introduction

2. Actual FDA-approved moAbs for cancer treatment

3. MoAbs under clinical development

4. Conclusion

5. Expert opinion

\section{informa} healthcare
Monoclonal antibodies for the treatment of non-haematological tumours: update of an expanding scenario

\author{
Giuseppe Bronte, Giovanni Sortino, Francesco Passiglia, Sergio Rizzo, \\ Francesca Lo Vullo, Antonio Galvano, Viviana Bazan, Christian Rolfo \& \\ Antonio Russo ${ }^{\dagger}$ \\ ${ }^{\dagger}$ University of Palermo, Department of Surgical, Oncological and Oral Sciences, Section of Medical \\ Oncology, Palermo, Italy
}

Introduction: The identification of cell membrane-bound molecules with a relevant role in cancer cell survival prompted the development of moAbs to block the related pathways. In the last few years, the number of approved moAbs for cancer treatment has constantly increased. Many of these drugs significantly improved the survival outcomes in patients with solid tumours.

Areas covered: In this review, all the FDA-approved moAbs in solid tumours have been described. This is an update of moAbs available for cancer treatment nowadays in comparison with the moAbs approved until few years ago. The moAbs under development are also discussed here.

Expert opinion: The research on cancer antigens as therapeutic targets led to an expanding scenario of available treatment options in non-haematological malignancies. In a few years, the number of approved drugs has increased rapidly. Some of these agents are actually on label in combination with standard chemotherapy. Only some of them can be delivered as monotherapy. The research on these new drugs is addressing both the identification of further target molecules in key cancer-related pathways and the improvement of drug effectiveness by changing the affinity and the selectivity of a moAb relative to its target.

Keywords: cancer, cancer antigen, cytotoxic T-lymphocyte antigen 4, EGFR, HER2, immunotherapy, moAbs, receptor activator of nuclear factor $\kappa \mathrm{B}$ ligand, VEGF

Expert Opin. Biol. Ther. (2015) 15(1):45-59

\section{Introduction}

The research on cancer immunology led to the development of moAbs targeting specific tumour antigen. First these molecules were used with the aim of eliciting an immunologic response against tumour cells. However, preclinical research prompted the use of moAbs to target these oncogenic pathways, which start their signalling through some molecules expressed on tumour cell surface.

Three years ago, we published a review about moAbs and antibody fragments in solid tumours. In that paper, only four approved moAbs for the treatment of solid tumours were reported [1]. Nowadays, the number of FDA-approved moAbs in solid tumours has doubled. And even greater is the number of those moAbs in clinical development for use in non-haematological malignancies. MoAbs have been produced by different techniques, such as mouse ascites fluid and tissue cultures. The application of recombinant DNA technology to hybridoma technology has allowed production moAbs with greater safety and efficacy through chimerisation 


\section{Article highlights.}

- The number of moAbs for the treatment of non-haematological tumours is rapidly increasing.

- The current FDA-approved moAbs may be divided into five categories: anti-HER2, anti-EGFR, anti-VEGF, anti-CTLA-4 and anti-RANKL.

- Most of the approved moAbs achieved significant improvement of overall survival by the combination with standard chemotherapy, that is, trastuzumab, pertuzumab, cetuximab, panitumumab, bevacizumab, ramucirumab and aflibercept. The other moAbs are delivered as monotherapy, that is, T-DM1, ipilimumab and denosumab.

- Predictive biomarkers are available for some approved moAbs, that is, RAS mutations for anti-EGFR moAbs in metastatic colorectal cancer patients and HER2 expression for anti-HER2 moAbs in breast cancer patients.

- New moAbs are currently under investigation in clinical trials, targeting both the already known and the emerging cancer-related molecular pathways, in order to improve the efficacy of the available treatments.

This box summarises key points contained in the article.

and humanisation. By this way, different forms of moAbs are available including murine, human, chimeric and humanised moAbs (Figure 1).

Generally, the use of moAbs aims to achieve various goals, such as the blockade of oncogenic pathways with subsequent effects on cell growth and apoptosis, the modulation of immune response against tumour cells and more recently moAbs conjugated with cytotoxic drugs used to be a vehicle of these drugs to cancer cells [2-4].

Even though moAbs have been developed to be more specific for tumour cells with respect to cytotoxic drugs, side effects can arise during the treatment with moAbs [5,6]. These adverse effects could be a consequence of the targeting of receptors in normal cells or the activation of general immune response.

Here, we review the moAbs approved for the clinical use in non-haematological malignancies and also those that are under development through the improvement of previous strategies or the search for new targets. This paper could represent an update of our previous paper [1] to show how the scenario of moAbs use has been really expanding.

\section{Actual FDA-approved moAbs for cancer treatment}

\subsection{Anti-HER2 (trastuzumab, T-DM1, pertuzumab) 2.1.1 Trastuzumab}

Human epidermal growth factor receptor 2 (HER2) is a receptor of the EGFR family, which has been found to be overexpressed in various tumour types but the most relevant clinical implications are today well known for breast, gastric and gastroesophageal junction cancers. HER2 protein overexpression found at immunohistochemistry (IHC) (IHC 3+) or CerbB-2 gene amplification at fluorescence in situ hybridisation (FISH) are known to be negative prognostic factors and a predictive for response to anti-HER2 drugs (trastuzumab, lapatinib, pertuzumab) in patients with breast cancers: almost $15-20 \%$ of them are HER2 positive [7]. Trastuzumab is a humanised moAb directed against the extracellular domain of the HER2/neu receptor. When this antibody binds its target, it inhibits the effect of the homodimerisation of HER2 and the cleavage of the receptor with the consequent blockade of the signal transduction cascade and inhibition of the proliferation and survival of HER2-dependent tumours. An antibody-dependent cell-mediated toxicity has been demonstrated to be one of the possible mechanisms of action [8]. Trastuzumab has been approved for HER2-positive breast cancer (in metastatic, neoadjuvant and adjuvant setting) and for the treatment of locally advanced and metastatic gastric and gastroesophageal junction cancer and its activity has been evaluated with various chemotherapeutic drugs such as taxanes and anthracyclines, platinum salts, fluorouracil, gemcitabine and vinca alkaloids. The use of trastuzumab in HER2-positive untreated metastatic patients showed a strong improvement in terms of time-to-progression and overall survival (OS) when administered in combination with chemotherapy in a pivotal Phase III trial [9]. These results have been confirmed by subsequent trials with taxanes [10,11] and other chemotherapeutic agents [12-16]. Trastuzumab has also been studied as monotherapy for metastatic breast cancer patients but combination regimens showed greater results in terms of responses [17]. Resistance to trastuzumab usually occurs within 1 year after the start of treatment [18] but after disease progression the continuation of trastuzumab in combination with a different subsequent chemotherapy regimen is a possible valid strategy, suggested by some evidences $[19,20]$.

Several studies in the adjuvant setting, enrolling $>10,000$ patients, evaluated the addition of trastuzumab to chemotherapy and in a meta-analysis of prospective trials, the combination, compared with chemotherapy alone, showed a significant benefit in terms of disease-free survival (odds ratio $[\mathrm{OR}]=0.69)$ and $\mathrm{OS}(\mathrm{OR}=0.78)$, with a reduction of local relapse by about $50 \%$ and distant recurrence near to $40 \%$. The improvements were demonstrated also when trastuzumab, after the completion of chemotherapy was given alone, in combination with taxanes following anthracyclines plus cyclophosphamide and in combination with carboplatin and docetaxel. These data confirmed the important role of trastuzumab in the adjuvant setting for HER2-positive patients with tumour diameter $>1 \mathrm{~cm}$ or metastatic lymph nodes [21]. Data for patients with HER2-positive tumours $(\leq 1 \mathrm{~cm})$ and negative lymph nodes are today controversial. Most of the studies, which evaluated the addition of trastuzumab to chemotherapy as adjuvant treatment, also enrolled node-negative patients but with tumours $>1 \mathrm{~cm}$ in diameter. This category of patients obtained significant benefits from 


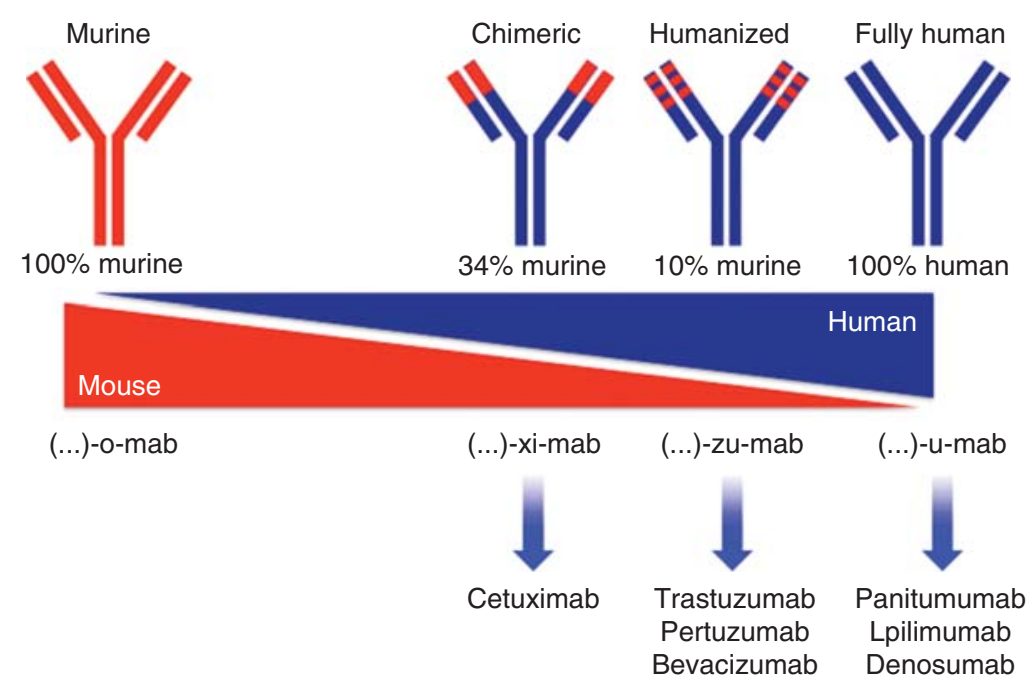

Figure 1. Classification of moAbs according to the proportion of murine and human peptidic sequences.

the addition of trastuzumab similar to those with late-stage HER2-positive tumours [22,23]. There are no prospective data for patients with HER2-positive node-negative and $<1 \mathrm{~cm}$ tumours. Retrospective studies indicate that these patients have a worse prognosis [24,25] (compared with those with HER2-negative tumours and the same diameter) and that they could benefit from the use of trastuzumab as adjuvant therapy. Today, this benefit is not clear and data are not always significant [26-28]. The duration of treatment with trastuzumab in the adjuvant setting is still a topic of discussion. At present, the duration of 1 year, after completion of chemotherapy, represents the standard of care $[22,29,30]$.

A recently approved indication of trastuzumab is for HER2-positive metastatic gastric and esophagogastric junction tumours. In fact, $\sim 20 \%$ of them overexpress HER2 and this represented the rationale to study trastuzumab in these types of tumours [31]. The addition of trastuzumab to HER2-positive gastric and gastroesophageal junction cancers, in a Phase III randomised trial (ToGA trial), showed an improvement in OS for patients in the trastuzumab plus chemotherapy arm versus chemotherapy alone (13.8 vs 11.1 months), obtaining a $26 \%$ of reduction of the death rate. This improvement has been obtained only for patients whose tumours were at IHC $3+$ or IHC $2+$ and FISH positive. No survival benefit has been shown in IHC 0 or $1+$ and FISH-positive patients [32]. Trastuzumab is now a very well-known drug, which has fully entered into the daily clinical practice, both for breast cancer and for metastatic gastric and esophagogastric junction cancers. It is a very well-tolerated drug, with an excellent profile of toxicity, but the only real limitation to its use is represented by cardiotoxicity. For this reason, although some data regarding safety with liposomal anthracyclines are available, the addition of trastuzumab to standard anthracyclines is not recommended $[33,34]$.

\subsubsection{T-DM1 (trastuzumab emtansine)}

Trastuzumab-DM1 (trastuzumab emtansine) is an antibodydrug conjugate composed of anti-HER2 moAb trastuzumab, linked to an anti-microtubule agent DM1, which is a derivate of maytansine [35]. The antitumour action of this drug is related not only to the well-known role of trastuzumab, but also to the inhibition of microtubular assembly induced by DM1 on HER2-overexpressing cells. Indeed, trastuzumab delivers DM1 to the targeted tumour cells, focusing its toxicity almost only on cancer cells [36]. Moreover, it seems that T-DM1 is internalised after binding cancer cells' surface receptors [37]. The drug has been approved in February 2013 by FDA for the treatment of HER2-positive metastatic breast cancer patients who have received prior trastuzumab and a taxane-based chemotherapy. After deriving promising data from Phase I and II studies [36,38-40], it has been tested in Phase III trials. In the EMILIA trial, a randomised Phase III study, T-DM1 has been evaluated in comparison with lapatinib plus capecitabine, in patients with HER2-positive locally advanced or metastatic breast cancer who had prior received treatment with trastuzumab and taxane-based chemotherapy. Nine hundred and ninety-one patients were enrolled and primary end points were progression-free survival (PFS) and OS. Results showed an improvement both in median PFS (9.6 vs 6.4 months; hazard ratio [HR]: 0.65; 95\% CI: $0.55-0.77 ; \mathrm{p}=0.001)$ and in median OS (30.9 vs 25.1 months; HR: 0.68; 95\% CI: 0.55 - 0.85; $\mathrm{p}=0.001)$ for patients in the T-DM1 arm. A higher response rate was seen in patients treated with T-DM1 (43.6 vs 30.8\%; $\mathrm{p}=0.001)$ and its toxicity profile was better than the combination lapatinib plus capecitabine, with a grade 3 event rates of 41 versus $57 \%$, respectively [41]. These positive data are particularly interesting as they were obtained in pre-treated patients regardless of the number of previous treatments. Moreover, a survival benefit was also seen in patients who 


\section{G. Bronte et al.}

had disease progression within 6 months following the completion of adjuvant or neoadjuvant treatment with trastuzumab. The MARIANNE trial, a randomised, three-arm Phase III study, is evaluating efficacy and safety of T-DM1 with pertuzumab or without pertuzumab, versus the combination of trastuzumab plus taxanes in HER2-positive patients with progressive or recurrent locally advanced or previously untreated metastatic breast cancer and primary end point is PFS. One thousand ninety-five patients have been enrolled to date and results are awaited [42]. T-DM1 has been tested, in the TH3RESA study, in comparison with treatment of the physician's choice in patients with metastatic or unresectable locally advanced/recurrent HER2-positive breast cancer, who have been treated at least with two prior HER2-directed therapies. Co-primary outcome measures of this Phase III trial are PFS and OS in the intention-to-treat population. In February 2013, 602 patients were enrolled and the 75\% of them had visceral disease. Patients had received a median of four prior regimens for the advanced disease. Results showed an improvement in median PFS of almost 3 months for patients receiving T-DM1 (6.2 vs 3.3 months; HR:0.528; 95\% CI: $0.422-0.661 ; \mathrm{p}<0.0001)$ while statistically significant data for OS had not yet been reached, although the interim analysis showed a positive trend for T-DM1 arm. Objective responses also improved for T-DM1 patients with a consistent difference (31.3 vs $8.6 \%$; $\mathrm{p}<0.001$ ) and crossing over from the control to the experimental arm was permitted after progression. Serious adverse events were fewer in the TDM1 arm than in the treatment of the physician's choice one [43]. These data, as already pointed out by the EMILIA trial and previous studies, confirm the great importance of T-DM1 in the panorama of treatments for patients with HER2-positive metastatic breast cancer.

\subsubsection{Pertuzumab}

Pertuzumab is a fully humanised moAb directed against the extracellular dimerisation domain II of HER2. It binds a different epitope of the HER2 receptor from the one targeted by trastuzumab [44]. In fact, trastuzumab and pertuzumab work with a complementary action because the first inhibits ligand-independent HER2 signalling without preventing ligand-activated HER2/HER3 or HER2/HER1 heterodimerisation, whereas pertuzumab prevents the formation of homoand heterodimers of HER2 with the other EGFRs, including HER1, HER3 and HER4. Both in vitro and in vivo preliminary evidences of trastuzumab's and pertuzumab's synergic actions have been available for some years and these data were conducted to test the two drugs together for clinical use $[45,46]$. Pertuzumab was the first drug approved by FDA in 2012, with the indication of first-line treatment in combination with trastuzumab and docetaxel for patients with HER2-positive metastatic breast cancer naïve to any antiHER2 therapy or chemotherapy for metastatic disease on the basis of the CLEOPATRA study. In this trial, 808 patients with HER2-positive metastatic breast cancer were randomised to receive a combination of trastuzumab and docetaxel with either placebo or pertuzumab as first-line treatment [47]. The study met its primary end point with an improvement of 6 months in PFS for patients in the pertuzumab arm (18.5 vs 12.4 months; HR: 0.62 ; $95 \% \mathrm{CI}$ : 0.51 to 0.75 ; $\mathrm{p}<0.001$. A further analysis showed a significant benefit also in OS for patients allocated in the pertuzumab group, although it had not been reached at the time of the analysis (95\% CI: 42.4-not available) and no differences in cardiotoxic effects were seen between the two groups [48,49]. In September 2013, a second indication was approved for pertuzumab on the basis of the results shown by the Phase II NeoSphere trial, in which 417 chemo-naïve patients with HER2-positive early-stage breast cancer were randomised in four arms to receive: trastuzumab plus docetaxel ( $\mathrm{n}=107$, group A), pertuzumab and trastuzumab plus docetaxel ( $\mathrm{n}=107$, group $\mathrm{B}$ ), pertuzumab plus trastuzumab ( $\mathrm{n}=107$, group C) or pertuzumab plus docetaxel $(\mathrm{n}=96$, group D), with pathological complete response in the breast as primary end point. Pertuzumab was administered at the loading dose of $840 \mathrm{mg}$, followed by $420 \mathrm{mg}$ every 3 weeks, trastuzumab first at $8 \mathrm{mg} / \mathrm{kg}$ loading dose, followed by $6 \mathrm{mg} / \mathrm{kg}$ every 3 weeks and docetaxel in the group A $75 \mathrm{mg} /$ $\mathrm{m}^{2}$, escalating, if tolerated, to $100 \mathrm{mg} / \mathrm{m}^{2}$ every 3 weeks. Patients in the pertuzumab and trastuzumab plus docetaxel showed a higher complete response rate (pCR: 45.8; 95\% CI: 36.1 - 55.7) compared with that obtained in the trastuzumab plus docetaxel group (pCR: 29; 95\% CI: 20.6 - 38.5). Results were inferior in the pertuzumab plus docetaxel arm (pCR: 24; 95\% CI: 15.8 - 33.7) and in the pertuzumab plus trastuzumab group (pCR: 16.8; 95\% CI: 10.3 - 25.3). The rates of severe adverse events were similar across the groups A, B and D, but obviously lower in group $\mathrm{C}$ [50]. This approval was supported also by the results of another Phase II study: the TRYPHAENA trial. This study was conducted to assess the toxicity of the anti-HER2 therapy with anthracyclines and carboplatin in patients with localised, inflammatory or early ( $2 \mathrm{~cm}$ tumour size or lymph node-positive) HER2-positive breast cancer. Patients were treated with anthracycline-containing chemotherapy administered with sequential or concurrent trastuzumab and pertuzumab or with carboplatin and docetaxel in addition to concurrent trastuzumab and pertuzumab. The highest $\mathrm{pCR}$ rate was obtained with the carboplatin-docetaxel regimen plus trastuzumab and pertuzumab (66\%). The addition of trastuzumab and pertuzumab to chemotherapy resulted in low rates of symptomatic left ventricular systolic dysfunction [51]. For these reasons, in the neoadjuvant setting, pertuzumab has to be administered at the initial loading dose of $840 \mathrm{mg}$ in a $60 \mathrm{~min}$ infusion followed by a continuation dose of $420 \mathrm{mg}$ intravenously (i.v.) as a $30-60$ min infusion repeated every 21 days for three or six cycles in addition to chemotherapy: for four preoperative cycles in combination with docetaxel and trastuzumab followed by three cycles of fluorouracil, epirubicin and cyclophosphamide (FEC), for three preoperative 
cycles in combination with docetaxel and trastuzumab after three FEC or for six preoperative cycles with docetaxel, carboplatin and trastuzumab.

\subsection{Anti-EGFR (cetuximab, panitumumab)}

EGFR represents today one of the most important targets for cancer therapy, especially for the treatment of metastatic colorectal cancer (mCRC) and head and neck cancers. Two moAbs directed against EGFR are available for cancer therapy: cetuximab and panitumumab. Cetuximab is a chimeric moAb that binds the extracellular domain of EGFR blocking its activation normally provided by EGF [52,53].

The drug has been approved by FDA in 2004 for the treatment of EGFR-expressing, $\mathrm{mCRC}$ in combination with irinotecan on the basis of the results of the BOND trial [54]. The EPIC trial confirmed the promising activity of cetuximab showing an advantage in terms of time-to-progression and response rate with the addition of the drug with irinotecan compared with irinotecan alone for mCRC patients after progression on 5-fluorouracil (5-FU) and oxaliplatin chemotherapy. No differences in OS were demonstrated probably for the great number of patients in the irinotecan arm who received cetuximab and irinotecan at progression [55]. In the CRYSTAL study, the addition of cetuximab to FOLFIRI produced positive results in terms of response rate (RR), PFS and OS, but these benefits were limited to KRAS wild-type patients. Moreover, the presence of $B R A F^{\mathrm{V} 600 \mathrm{E}}$ mutation conferred a poorer prognosis in both the arms [56-58]. The positive results in terms of PFS of the combination of cetuximab with oxaliplatin-based chemotherapy in the OPUS trial, in KRAS wild-type patients, led to the approval of cetuximab as firstline treatment in EGFR-expressing and KRAS wild-type mCRC patients [59]. In contrast to these data, both in the COIN and the Nordic FLOX trial the addition of cetuximab to either FOLFOX/Capecitabine plus oxaliplatin (CAPOX) (MRC COIN) or oxaliplatin plus a fluoro-pyrimidine (FLOX) (NordicFLOX) did not demonstrate a significant improvement in either OS or PFS. A deeper analysis of the COIN study showed differences between the results obtained with the use of i.v. 5-FU or capecitabine in combination with oxaliplatin and cetuximab, and particularly bad data emerged when cetuximab and oxaliplatin were associated with capecitabine. Moreover, the bolus administration of 5-FU provided in the NORDIC trial seems to have a detrimental effect with the addition of oxaliplatin and cetuximab [60,61]. Recently, a rechallenge with cetuximab in pre-treated mCRC patients have shown a further benefit. By this finding, a model for the development of resistance to cetuximab has been proposed [62].

The improvements in OS obtained with the addition of cetuximab to radiotherapy compared with radiotherapy alone in a Phase III randomised trial led to FDA approval of cetuximab for head and neck cancers for use in combination with radiation therapy for the treatment of locally or regionally advanced squamous cell carcinoma and as a single agent for the treatment of patients with recurrent or metastatic squamous cell carcinoma of the head and neck after platinumbased therapy failure [63]. Finally, on the basis of the positive results of a Phase III trial, FDA approved the use of cetuximab in combination with platinum- and 5-FU-based chemotherapy as first-line therapy for recurrent or metastatic patients affected by squamous cell carcinoma of the head and neck [64].

The second moAb available for the treatment of $\mathrm{mCRC}$ is panitumumab, a fully human moAb approved in 2006 for patients with EGFR-expressing mCRC after progression or following fluoropyrimidine-, oxaliplatin- and irinotecancontaining regimens. The efficacy of the drug in monotherapy has been demonstrated in comparison with best supportive care (BSC) in $463 \mathrm{mCRC}$ patients refractory to 5-FU, irinotecan and oxaliplatin. In this trial, although no improvement in OS was obtained, panitumumab provided a significant benefit in RR ( 10 vs $0 \%$; p < 0.001 ) and PFS at 8 weeks (49 vs 30\%; p < 0.001) and further analysis confined these benefits to KRAS wild-type patients [65,66]. The use of panitumumab as first-line treatment in addition to FOLFOX chemotherapy has been evaluated in the randomised PRIME trial, the results of which showed an improvement both in median PFS for KRAS wild-type mCRC patients treated with panitumumab plus chemotherapy (10 vs 8.6 months; HR: $0.80 ; \mathrm{p}=0.01)$ and in median OS (23.9 vs 19.7 months; HR: $0.88 ; p=0.17)[67]$. A recent retrospective analysis conducted on the samples of the PRIME trial demonstrated that other KRAS mutations in exons 3 and 4 (in addition to the well-known exon 2 mutations) and NRAS mutations (on exons 2, 3 and 4) can determine resistance to panitumumab and that the use of the drug is associated with a detrimental effect in mutated patients [68]. On the basis of these criteria, the use of panitumumab is approved only for RAS (KRAS and NRAS) wild-type mCRC patients. Moreover, a retrospective analysis of the OPUS trial led to similar conclusions when patients with mutations in exons 2, 3 and 4 of NRAS and in exons 3 and 4 of $K R A S$ were excluded from the exon 2 $K R A S$ wild-type population. For these reasons, the limitation of the use of panitumumab in KRAS and NRAS wild-type patients has been extended also to the use of cetuximab. In the second-line setting, panitumumab has been tested in combination with FOLIFIRI versus FOLFIRI alone in KRAS wild-type patients. This trial demonstrated an improvement in PFS for patients treated with the combination (6.7 vs 4.9 months; HR: $0.82 ; \mathrm{p}=0.0023$ ) and a trend toward longer OS but without statistical significance [69]. The need for a more precise selection of $\mathrm{mCRC}$ patient candidates for antiEGFR treatment has been confirmed by the analysis of the results of the FIRE-3 trial, in which 592 KRAS wild-type mCRC patients were randomised to receive FOLFIRI plus either cetuximab or bevacizumab as first-line treatment. The results showed an improvement in median OS of almost 4 months in the cetuximab arm but no benefit in terms of overall response rate (ORR) and PFS was observed when considering only exon $2 K R A S$ wild-type tumours [70]. Moreover, 


\section{G. Bronte et al.}

an updated analysis of the Phase III CRYSTAL study, presented at the 2014 American Society of Clinical Oncology (ASCO) Annual Meeting by Professor Ciardiello, demonstrates that the addition of cetuximab to FOLFIRI as the first-line treatment in mCRC provides a significant benefit across all end points in RAS wild-type (KRAS and NRAS wild-type) patients, whereas in patients with any tumour with $R A S$ mutation, no benefit was found [71].

\subsection{Anti-VEGF (bevacizumab)}

Bevacizumab, another cornerstone of anticancer therapy, is a humanised moAb that inhibits angiogenesis through the binding of VEGF, which is one the most important mediators of neo-angiogenesis and tumour growth. The development of a new tumour vascularisation mediated by VEGF and other growth factors induced through the hypoxia-inducible transcription factor is in fact considered a key target to inhibit tumour proliferation [72,73]. Although four different isoforms of VEGF have been identified (VEGF-A, -B, -C, -D), VEGF-A is considered the most important and its functions are mediated by the interaction with VEGF receptors (VEGFR1 or Flt-1, and VEGFR2 or KDR) [74,75]. Bevacizu$\mathrm{mab}$ is able to bind all isoforms of VEGF preventing the interaction with its receptors and their subsequent activation [76]. It has also been demonstrated that VEGFR inhibition mediated by bevacizumab on cancer cells induces a regression of abnormal tumour vascularisation with the normalisation of the vascular bed and the inhibition of neo-angiogenesis [77]. The most common adverse events related to bevacizumab showed in all clinical trials are hypertension, bleeding and proteinuria. Although a frequent blood pressure control is recommended, hypertension is often manageable with oral antihypertensives. During the last 10 years, bevacizumab has been studied for various diseases reaching many indications for solid cancers such as mCRC, metastatic breast cancer, metastatic renal cell carcinoma, metastatic ovarian cancer, advanced NSCLC and glioblastoma. After first approval of bevacizumab as first-line treatment for patients with mCRC in 2004 on the basis of previous positive studies with both 5-FU/leucovorin in a Phase II trial [78] and with IFL-Saltz regimen [79], other trials evaluated the role of bevacizumab in addition to oxaliplatinbased regimens as first-line treatment of $\mathrm{mCRC}$ or in addition to capecitabine monotherapy, but they did not reach the same statistical significance [80]. To date, bevacizumab is approved as first- and second-line treatment of mCRC patients in addition to 5-fluorouracil-irinotecan- or 5-fluorouracil-oxaliplatin-based chemotherapy [81-83]. In 2013, FDA approved bevacizumab in combination with fluoropyrimidine-irinotecan- or fluoropyrimidine-oxaliplatin-based chemotherapy for the treatment of patients whose disease has progressed during the first-line treatment with a bevacizumab-containing regimen. The rationale of 'bevacizumab beyond progression' came from the results of the ML18147 Phase III trial, in which 820 patients previously treated with bevacizumab-containing regimens were randomised to receive chemotherapy alone $(\mathrm{n}=411)$ or chemotherapy in combination with bevacizumab $(n=409)$. Then bevacizumab was administered until disease progression or unacceptable toxicity. The addition of bevacizumab to chemotherapy provided a small but statistically significant improvement in median OS (11.2 vs 9.8 months; HR: $0.81 ; \mathrm{p}=0.0062)$ [84]. A subsequent analysis of the trial confirmed these positive data, which seem to be independent of the KRAS status [85]. Recently at the 2014 ASCO Annual Meeting, data about an alternative schedule of association of bevacizumab have been presented. The TRIBE trial evaluated efficacy and toxic impact of the addition of bevacizumab with FOLFIRINOX (5-fluorouracil, leucovorin, oxaliplatin and irinotecan) chemotherapy as first-line treatment of mCRC patients. This study demonstrated improvements in median PFS and median OS with FOLFIRI + bevacizumab versus FOLFIRI + bevacizumab independently of RAS and BRAF status, with a trend toward a larger benefit in BRAFmutated patients limited by small number of patients. This intensive treatment is also more toxic than standard treatments and is not suitable for all patients; it could be a good therapeutic option for a selected category of young and fit patients [86].

In 2006, on the basis of a large Phase III trial, the use of bevacizumab was approved as first-line therapy in combination with chemotherapy for patients with unresectable, locally advanced, recurrent or metastatic, non-squamous, NSCLC [87]. This limitation is linked to the higher risk of hemoptoe for squamous NSCLCs, which usually presents cavitated lesions involving the main bronchus and mediastinum great vessels as demonstrated by clinical trials [88]. These positive data of efficacy have been confirmed in the AVAiL trial, in which bevacizumab was administered with different platinum-based chemotherapy (cisplatin plus gemcitabine) in patients with stage IIIB/IV NSCLC [89]. The use of bevacizumab as first-line therapy for HER2-negative metastatic breast cancer patients in combination with paclitaxel received a rapid approval in 2008 on the basis of the positive data in terms of PFS and ORR in the interim analysis of the Phase III E2100 trial [90], but this accelerated approval was revoked in 2011 by FDA on the basis of the lack of benefit in OS in two subsequent trials: AVADO and RIBBON-1 [91,92]. Despite these conflicting data and the withdrawal of indication by the FDA, the European Medicines Agency (EMA) has still maintained bevacizumab's approval for HER2-negative metastatic breast cancer patients.

In 2009, FDA approved the use of bevacizumab in combination with IFN- $\alpha$ for the treatment of patients with metastatic renal cell carcinoma on the basis of the results of two randomised trials, which demonstrated advantages in terms of median PFS and ORR [93,94], whereas no benefit in OS was found [95]. In 2009, bevacizumab received another rapid approval as a single-agent treatment for patients with glioblastoma, after progression following prior therapy, on the basis of the positive data of two trials. In a Phase II trial (BRAIN), 167 patients were randomised to receive bevacizumab alone or bevacizumab in combination with irinotecan. Objective 
response rates were $28.2 \%$ for bevacizumab and $37.8 \%$ for the combination arm, respectively. Median 6-month survival was $42.6 \%$ for bevacizumab and $50.3 \%$ for the combination arm and median OS was 9.2 and 8.7 months, respectively [96]. These results have been confirmed by a NCI Phase II study, in which 48 heavily pre-treated patients with recurrent glioblastoma have been treated with bevacizumab monotherapy and have received irinotecan in combination after disease progression. The study showed a 6-month PFS of $29 \%$ with a median OS of 31 weeks and a $35 \%$ of objective responses [97]. EMA did not approve this indication of bevacizumab probably for the lack of consistent data. The results of two randomised trials have led to the European approval of bevacizumab for the treatment of advanced ovarian cancer. In these two studies (GOG218 and ICON7), the addition of bevacizumab to standard carboplatin-paclitaxel chemotherapy followed by bevacizumab maintenance has been compared with chemotherapy alone obtaining an improvement in PFS (18.2 vs 12.7 months in the GOG0218, 18.3 vs 16.0 in the ICON7) [98]. In the planned final analysis of the ICON7 trial, presented at the 2013 European Cancer Congress, a 4.8-month benefit in OS was demonstrated in patients with bad prognosis and treated with bevacizumab plus chemotherapy [99].

\subsection{Anti-VEGFR (ramucirumab, aflibercept)}

Ramucirumab is a new human recombinant moAb of the IgG1 class that binds to VEGFR2 and blocks the activation of the receptor. It has been recently approved by FDA as single agent for the treatment of patients with advanced or metastatic gastric or gastroesophageal junction adenocarcinoma with disease progression on or after prior treatment with fluoropyrimidine- or platinum-containing chemotherapy. This approval was supported by the positive results of the two randomised, Phase III, REGARD and RAINBOW studies, comparing respectively ramucirumab plus BSC versus placebo plus BSC, and ramucirumab in combination with paclitaxel versus paclitaxel plus BSC, in patients with pretreated advanced gastric or gastroesophageal junction adenocarcinoma. Both these trials have shown that the addiction of ramucirumab significantly improved the patients' survival outcomes: OS (REGARD: 5.2 vs 3.8 months; HR: 0.776; RAIMBOW: 9.63 vs 7.36 months; HR: 0.87) and PFS (REGARD: 2.1 vs 1.3 months; HR: 0.483; RAIMBOW: 4.40 vs 2.86 months; HR: 0.635) [100,101], with an acceptable toxicity profile. The most common adverse events observed in ramucirumab-treated patients were hypertension and diarrhoea. The grade 3 - 4 adverse reactions included hypertension and hyponatremia. This new moAb is also under clinical investigation in several Phase III trials of colorectal cancer (NCT01183780), hepatocellular carcinoma (NCT0 1140347), NSCLC (NCT01168973) and breast cancer (NCT00703326). Promising results from these studies are expected within the end of 2014.

Aflibercept is a fusion protein consisting of portions of human VEGFR1 - 2 extracellular domains fused to the crystallisable fragment portion of human $\operatorname{IgG} 1$, produced by recombinant DNA technology. Although it is not an antibody itself, it deserves to be mentioned among the new approved treatments targeting the angiogenesis process. It has been evaluated in combination with irinotecan-based chemotherapy in a Phase III randomised study in patients with mCRC refractory to oxaliplatin-based chemotherapy. The addition of aflibercept to FOLFIRI regimen significantly improved OS (13.5 vs 12.06 months; HR: 0.817), PFS (6.90 vs 4.67 months; HR: 0.758$)$ and RR (19.8 vs $11.1 \%$ : p = 0.0001), with an acceptable toxicity profile [102]. The positive results of the VELOUR trial led to the clinical approval of aflibercept as the second-line therapy in patients with mCRC, who have progressed to an oxaliplatin-based first-line therapy independent from prior use of bevacizumab.

\subsection{Anti-CTLA-4 (ipilimumab)}

In 2011, ipilimumab was approved by the FDA for the treatment of patients with unresectable or metastatic melanoma, who had previously been treated with standard chemotherapy. Ipilimumab is a fully human moAb directed against CTLA-4 receptor, which is located on the surface of activated $\mathrm{T}$ lymphocytes. Normally, the interaction between CTLA-4 and its ligands (CD80/CD86), expressed on antigen-presenting cells, produces an inhibitory signal within the lymphocyte contributing to the homeostatic regulation of the immune response. Ipilimumab binds CTLA-4, thus blocking its activation and finally producing an immune-stimulating effect on $\mathrm{T}$ lymphocytes [103]. Ipilimumab approval was based on the results of a randomised three-arm Phase III trial, in which pre-treated 676 patients with advanced melanoma were randomised to receive ipilimumab at the dose of $3 \mathrm{mg} / \mathrm{kg}$ in combination with a gp100 peptide vaccine ( $\mathrm{n}=403)$, or ipilimumab $3 \mathrm{mg} / \mathrm{kg}$ plus placebo $(\mathrm{n}=137)$, or only gp100 vaccine $(n=136)$. Median OS was significantly longer for patients treated with ipilimumab alone (10.1 vs 6.4 months; HR: 0.66; $\mathrm{p}=0.003)$ or administered in combination with gp100 (10.0 vs 6.4 months; HR: 0.68; p < 0.001) compared with patients treated with gp100 alone. In this study, almost $15 \%$ of the patients showed grade 3 and 4 adverse events and the most frequent immune-related adverse event was diarrhoea. Seven immune-related deaths were registered [104]. A second Phase III trial evaluated ipilimumab in 502 previously untreated patients with metastatic melanoma. Patients were randomised to receive ipilimumab $(10 \mathrm{mg} / \mathrm{kg}$ every 21 days for four times as induction, and then $10 \mathrm{mg} / \mathrm{kg}$ every 3 months in maintenance) in combination with dacarbazine or dacarbazine plus placebo. The primary end point of the study was achieved with a significant improvement in OS for patients receiving ipilimumab plus dacarbazine (11.2 vs 9.1 months; HR: 0.72; $<<0.001$ ) and with a higher 3-year survival rate (20.8 vs $12.2 \%)$. The combination of dacarbazine with ipilimumab increased liver toxicity and grade $3-4$ adverse events occurred in $56.3 \%$ of the patients in the ipilimumab plus dacarbazine group. No toxic death was 


\section{G. Bronte et al.}

registered [105]. A promising field of research on ipilimumab is represented by lung cancer [106,107]. Two Phase III trials have evaluated ipilimumab in both advanced NSCLC [108] and extensive-stage SCLC [109], showing very promising results.

\subsection{Anti-receptor activator of nuclear factor kappaB ligand (denosumab)}

One of the most frequent sites of metastasis of solid tumours is the bone and the presence of bone metastasis, although often not considered a life-threatening condition, modifies greatly patients' quality of life and prognosis because it may cause complications such as fractures, severe pain, nerve compression and hypercalcaemia. Malignant cells derived from the primary tumour once located in the bone are able to induce the production by osteoblasts of receptor activator of nuclear factor $\kappa \mathrm{B}$ ligand (RANKL) through the secretion of many cytokines and growth factors. The interaction between RANKL and its receptor RANK leads to an osteoclastic activation and thus to a disequilibrium between bone formation and resorption [110]. Denosumab is a fully human moAb that binds with high affinity Rank-ligand (RANKL) preventing its interaction with RANK. The decreased availability of complex RANKL/RANK reduces the number and functions of osteoclasts, decreasing bone resorption and destruction induced by malignant cells. It was approved by FDA in 2010 for the prevention of skeletal-related events in patients with bone metastases from solid tumours on the basis of three randomised trials in which denosumab, administered subcutaneously at the dose of 120 every 4 weeks, has been compared with zoledronic acid $4 \mathrm{mg}$ administered i.v. every 4 weeks. In these studies, patients previously treated with i.v. bisphosphonate and with reduced renal function (creatinine clearances $<30 \mathrm{ml} / \mathrm{min}$ ) were excluded. In all these trials, denosumab resulted superior to zoledronic acid in preventing or delaying skeletal complications in patients with advanced malignancies and bone metastases, although no improvement in OS was observed. The most severe clinical problems linked to denosumab and highlighted by the cited studies are: hypocalcaemia and osteonecrosis of the jaw, which occurred in a higher percentage of patients treated with denosumab (3.1\%) than those treated with zoledronic acid (1.3\%) [111]. For these reasons, it is mandatory to perform an oral examination before starting denosumab and to administer an adequate supplement of calcium and vitamin D to all candidate patients. In another randomised, Phase III, placebo-controlled trial, denosumab was studied to evaluate if the treatment of patients with non-metastatic castration-resistant prostate cancer could prevent or delay the occurrence of bone metastasis. Results showed that denosumab significantly prolonged bone metastasis-free survival compared with placebo (29.5 vs 25.2 months; HR: $0.85 ; \mathrm{p}=0.028)$ and delayed time to first bone metastasis (33.2 vs 29.5 months; HR: 0.84; $\mathrm{p}=0.032$ ), whereas no improvement in OS was obtained [112]. These interesting data suggest that although not providing benefits in terms of survival, the use of drugs that target the bone microenvironment through RANK functions could create hostile conditions for the development of bone metastasis. Finally, on 24 July 2014, denosumab received an extended indication from the EMA Committee for Medicinal Products for Human Use, concerning the treatment of adults and skeletally mature adolescents with giant cell tumour of bone that is unresectable or where surgical resection is likely to result in severe morbidity.

\section{MoAbs under clinical development}

Since the approval of the first moAb, hundreds of moAbs, including murine, chimeric and humanised antibodies, have been developed for cancer treatment. As reported here, some of these have been approved by FDA and are already available for clinical use in everyday practice, as monotherapy or in combination with standard chemotherapy regimens. However, most are actually under investigation in clinical trials, often in early clinical development (Table 1). Some of these new moAbs focus on molecular pathways, which have already been known to be related to cancer development and progression, trying to improve the efficacy of the available treatments. Other moAbs target the new emerging molecular pathways, which seem to be involved in carcinogenesis.

\subsection{The improvement of previous strategies}

Among the moAbs that are actually undergoing evaluation as cancer treatment in Phase III clinical studies, necitumumab, a recombinant human anti-EGFR moAb, which was designed to block the ligand binding site of the human EGFR was investigated in two randomised Phase III trials on NSCLC. The Phase III SQUIRE trial of gemcitabine-cisplatin chemotherapy plus necitumumab versus gemcitabine-cisplatin chemotherapy alone in the first-line treatment of patients with stage IV squamous NSCLC has been recently completed, showing that the addition of necitumumab to chemotherapy statistically significantly improved OS (HR: 0.84; $\mathrm{p}=0.012$ ) and PFS (HR: 0.85; $\mathrm{p}=0.020)$ [113]. Otherwise, the randomised Phase III trial (INSPIRE) of necitumumab plus cisplatinum-pemetrexed versus cisplatin-pemetrexed alone, as first-line therapy, in stage IV non-squamous NSCLC, did not meet its primary end point (PFS: 5.6 vs 5.6 months; HR: 0.96), resulting in a higher frequency of grade $\geq 3$ adverse events (skin reaction, gastrointestinal reaction, asthenia, etc.) [114]. Tremelimumab is an investigational, fully human moAb directed against CTLA-4, a co-inhibitory receptor that represses effector $\mathrm{T}$-cell activity in cancer. On the basis of promising responses observed in early clinical trials, a Phase III trial compared tremelimumab monotherapy versus standard chemotherapy as first-line therapy for advanced melanoma patients. Unfortunately, the study was stopped early, because no significant survival differences were observed between the two treatment arms [115]. Tremelimumab is also undergoing clinical investigation in several 
Table 1. New moAbs under clinical development.

\begin{tabular}{|c|c|c|c|}
\hline moAbs & Targets & $\begin{array}{c}\text { Solid } \\
\text { tumours }\end{array}$ & Ref. \\
\hline Ramucirumab & Anti-VEGFR2 & $\begin{array}{l}\text { CRC } \\
\text { HCC } \\
\text { NSCLC } \\
B C\end{array}$ & $\begin{array}{l}\text { NCT01183780 } \\
\text { NCT01140347 } \\
\text { NCT01168973 } \\
\text { NCT00703326 }\end{array}$ \\
\hline Necitumumab & Anti-EGFR & $\begin{array}{l}\text { NSCLC } \\
\text { (Squ) } \\
\text { NSCLC } \\
\text { (No-Squ) }\end{array}$ & $\begin{array}{l}{[113]} \\
{[114]}\end{array}$ \\
\hline Ipilimumab & Anti-CTLA-4 & $\begin{array}{l}\text { SCLC } \\
\text { NSCLC }\end{array}$ & $\begin{array}{l}{[107]} \\
{[108]}\end{array}$ \\
\hline Tremelimumab & Anti-CTLA-4 & $\begin{array}{l}\text { Melanoma } \\
\text { Mesothelioma } \\
\text { CRC } \\
\text { HCC }\end{array}$ & $\begin{array}{l}{[15]} \\
\text { NCT01843374 } \\
\text { NCT00313794 } \\
\text { NCT01008358 }\end{array}$ \\
\hline Nivolumab & Anti-PD-1 & Melanoma & $\begin{array}{l}\text { NCT02041533, } \\
\text { NCT01642004 } \\
\text { NCT01673867 } \\
\text { NCT01721772, } \\
\text { NCT01721746, } \\
\text { NCT01844505 } \\
\text { NCT01668784 }\end{array}$ \\
\hline MPDL3280A & Anti-PD-L1 & NSCLC & NCT02008227 \\
\hline Onartuzumab & Anti-cMet & NSCLC & {$[118,119]$} \\
\hline Rilotumumab & Anti-HGF & GC & NCT01697072 \\
\hline Farletuzumab & Anti-HFR & $\begin{array}{l}\text { OC } \\
\text { NSCLC }\end{array}$ & $\begin{array}{l}\text { NCT00849667 } \\
\text { NCT01218516 }\end{array}$ \\
\hline Racotumomab & $\begin{array}{l}\text { NGcGM3 } \\
\text { ganglioside } \\
\text { vaccine }\end{array}$ & NSCLC & NCT01460472 \\
\hline
\end{tabular}

BC: Breast cancer; CRC: Colorectal cancer; CTLA-4: Cytotoxic T-lymphocyte antigen 4; GC: Gastric cancer; HCC: Hepatocellular carcinoma; HFR: Human folate receptor; HGF: Hepatocyte growth factor receptor; OC: Ovarian cancer; PD-1: Programmed cell death 1; PD-L1: Programmed cell death-ligand 1; RCC: Renal cell carcinoma; Squ: Squamous.

Phase II trials of mesothelioma (NCT01843374), colorectal cancer (NCT00313794) and hepatocellular carcinoma (NCT01008358).

\subsection{The search for new targets}

Following the success of ipilimumab in the clinic, new antibodies against the immune-checkpoints inhibitors have been developed. The anti-programmed cell death 1 (PD-1) (nivolumab) and anti-programmed cell death-ligand 1 (PD-L1) (MPDL $3280 \mathrm{~A})$ are the two moAbs in the most advanced phases of clinical investigation. Both the new moAbs have shown a great activity in early clinical trials, showing great, durable responses in a variety of solid tumours, including NSCLC, castrationresistant prostate cancer, renal cell carcinoma, melanoma, $\mathrm{CRC}$ and gastric cancer $[116,117]$. On the basis of these promising results, nivolumab is actually undergoing clinical evaluation in several Phase III randomised trials, both as single agent, and in combination with other target therapies or chemotherapies, in NSCLC (NCT02041533, NCT01642004, NCT0167 3867), melanoma (NCT01721772, NCT01721746, NCT01
844505) and renal-cell carcinoma (NCT01668784). MPDL $3280 \mathrm{~A}$ is also under investigation in a randomised Phase III trial comparing anti-PD-L1 with docetaxel in patients with advanced NSCLC who have failed platinum therapy (NCT0 2008227). MetMab (onartuzumab) and AMG-102 (rilotumumab) are the two anti-cMet moAbs currently most advanced in clinical development. Onartuzumab is a humanised, monovalent antibody that blocks the ligand-binding site of the hepatocyte growth factor receptor, thereby inhibiting the MET dimerisation and the downstream signalling pathways. Despite the promising results of the randomised Phase II study [118], the subsequent Phase III trials did not confirm the efficacy results, showing that the addition of onartuzumab to erlotinib did not improve OS (HR: 1.27; $\mathrm{p}=0.068$; median OS: 6.8 vs 9.1 months), PFS (HR: 0.99; $\mathrm{p}=0.92$; median PFS: 2.7 vs 2.6 months) or ORR (8.4 vs $9.6 \%$; p = 0.63) [119]. Rilotumumab is a fully human moAb targeting human hepatocyte growth factor/scatter factor (HGF/SF), which inhibits the binding of HGF/SF to its receptor MET. Rilotumumab is undergoing clinical evaluation in a Phase III, randomised, placebo-controlled study, in combination with epirubicin, cisplatin and capecitabine as first-line therapy, in advanced, MET-positive, gastric or gastroesophageal junction adenocarcinoma (NCT01697072). Unfortunately, negative results emerged from the randomised, Phase III study of farletuzumab, a humanised moAb targeting human folate receptor, in combination with carboplatin and a taxane in patients with platinum-sensitive epithelial ovarian cancer, in first relapse (NCT008 49667). The study did not meet its primary end point PFS, but the drug is actually undergoing clinical investigation in lung cancer (NCT01218516). Finally, racotumomab is a murine moAb that mimics NGcGM3 ganglioside, highly expressed on the cell surface of several human cancers, including lung, breast, melanocytes and colon. Therefore, it acts as a therapeutic vaccine, triggering a specific immune response of the patient's immune system, against this specific target. A randomised, Phase III trial is ongoing, evaluating the efficacy and safety of active specific immunotherapy with racotumomab plus BSC versus BSC in patients with advanced NSCLC, who have not progressed during the standard first-line treatment (NCT01460472).

\section{Conclusion}

Since 3 years ago, the approval of moAbs for the treatment of non-haematological tumours has expanded rapidly. Nowadays, the number of moAbs approved for this label has doubled. These molecules can be grouped into five types: anti-HER2, anti-EGFR, anti-VEGF, anti-CTLA-4 and anti-RANKL.

All these drugs are supported by a solid basis of scientific evidences from clinical trials. The mechanisms of action, the outcomes and the toxicity profiles of these drugs are discussed here. Most of these moAbs achieved significant improvement of overall survival by the combination with standard 
Table 2. Approved moAbs in advanced solid tumours.

\begin{tabular}{|c|c|c|c|c|c|}
\hline Targets & moAbs & Solid tumours & Setting & OS (months) & Ref. \\
\hline \multirow[t]{8}{*}{ VEGF } & Bevacizumab & CRC & 1-line & 21.3 & {$[80,83,84]$} \\
\hline & & & 2-line & 12.9 & \\
\hline & & & Beyond PD & 11.2 & \\
\hline & & $B C$ & 1 -line & N.A. & {$[91,92]$} \\
\hline & & OC & 1-line & $39.3^{*}$ & [99] \\
\hline & & Glioblastoma & Pre-treated pts. & 9.2 & [96] \\
\hline & & $\mathrm{RCC}$ & 1-line & N.A. & [95] \\
\hline & & NSCLC & 1-line & 12.3 & {$[87]$} \\
\hline \multirow[t]{2}{*}{ VEGFR2 } & Ramucirumab & $\mathrm{GC}$ & 2-line & 5.2 & {$[100,101]$} \\
\hline & & & & 9.63 & \\
\hline VEGFR1/2 & Aflibercept & CRC & 2-line & 13.5 & {$[102]$} \\
\hline \multirow[t]{6}{*}{ EGFR } & Cetuximab & CRC & 1-line (RAS-WT) & & [71] \\
\hline & & & & 28.4 & \\
\hline & & $H \& N$ & Pre-treated (+RT) & 49.0 & {$[63,64]$} \\
\hline & & & 1-line $(+C T)$ & 10.1 & \\
\hline & Panitumumab & CRC & 1-line (RAS-WT) & 26.0 & {$[68,69]$} \\
\hline & & & 2-line (KRAS-WT) & N.A. & \\
\hline \multirow[t]{4}{*}{ HER2 } & Trastuzumab & $\mathrm{HER} 2+\mathrm{BC}$ & 1-line & 31.2 & [10] \\
\hline & & $\mathrm{HER} 2+\mathrm{GC}$ & 1-line & 13.8 & [32] \\
\hline & T-DM1 & $\mathrm{HER} 2+\mathrm{BC}$ & Pre-treated pts. & 30.9 & [41] \\
\hline & Pertuzumab & $\mathrm{HER} 2+\mathrm{BC}$ & 1-line & N.A. & [49] \\
\hline \multirow[t]{2}{*}{ CTLA-4 } & Ipilimumab & Melanoma & Unresectable or metastatic & 10.1 & {$[104,105]$} \\
\hline & & & & 11.2 & \\
\hline RANKL & Denosumab & Bone metastases & - & N.A. & {$[111]$} \\
\hline
\end{tabular}

*Poor prognosis pts.

BC: Breast cancer; CRC: Colorectal cancer; CT: Chemotherapy; GC: Gastric cancer; CTLA-4: Cytotoxic T-lymphocyte antigen 4; H\&N: Head and neck cancer; N.A: Not available; OC: Ovarian cancer; pts: Patients; RANKL: Receptor activator of nuclear factor $\kappa B$ ligand; RCC: Renal cell carcinoma; RT: Radiotherapy; WT: Wild-type.

chemotherapy, that is, trastuzumab, pertuzumab, cetuximab, panitumumab, bevacizumab, ramucirumab and aflibercept. The other moAbs are delivered as monotherapy, that is, TDM1, ipilimumab and denosumab (Table 2). For some of the approved moAbs, predictive biomarkers are available for the selection of those patients who could achieve the best outcomes. These biomarkers include RAS mutations for anti-EGFR moAbs in mCRC patients and HER2 expression for anti-HER2 moAbs in breast cancer patients. This review can provide a summary of new data available for all FDAapproved moAbs patients with solid tumours.

\section{Expert opinion}

This update shows that the research on moAbs targeting cancer, namely solid malignancies, is very rapid. These moAbs allow targeting both the key pathways of cancer cell survival and the molecules produced by stromal cells and immune cells in tumour microenvironment. These mechanisms of action led to an improvement of overall survival in comparison with the use of standard chemotherapy only.

Originally, there was a great expectation from these targeted drugs, which were deemed 'magic bullets' to definitively defeat cancer without the chemotherapy-related life-threatening toxicity. Nowadays, we know that the goals obtained by these moAbs are less striking than expected. Indeed, some of these drugs allowed an improvement of survival outcomes by just few months. This goal is not always clinically relevant even though statistically significant. In the meantime, the introduction of these moAbs into the clinical practice consistently increased the total outlay for cancer treatment.

This effect implies that not all the healthcare systems of various countries can afford these increasing costs, although FDA and other regulatory agencies approve these drugs on the basis of the scientific evidences from clinical trials. For these reasons, the applicability of new treatments in clinical practice has to be validated also on the basis of pharmacoeconomic analyses. The approval of further moAbs should be taken into account if their use is really affordable by healthcare systems.

The research on moAbs for solid malignancies is now addressing new targets. And also new approaches have been developed, such as the combination of a moAb with a cytotoxic drug in the same molecule. This strategy allows to make easier the delivery of a cancer treatment with the concomitant improvement of efficacy and the reduction of toxicity rates in comparison with the standard combination of a chemotherapy regimen together with a moAb.

The study of this kind of strategies would be optimised by the evaluation of targeted action on tumour tissue through molecular imaging, that is, positron-emission tomography. A similar result could be offered by the application of liquid 
biopsy, namely circulating tumour DNA, to monitor the clearance of pre-existing somatic mutations as a surrogate end point for the efficacy of moAbs.

We think that in this field of research, the most interesting area is represented by the finding of predictive biomarkers to select those patients with higher probability to respond to moAbs. These biomarkers need to be validated in large clinical trials before their use in clinical practice. This procedure of validation is possible for cancers with higher prevalence, that is, colorectal cancer and breast cancer. However, this goal is more difficult to achieve in other malignancies. So the research in these cases would require much more time.

The identification of valid biomarkers for patients' selection could be the proper solution to optimise the efficacy of a new treatment and subsequently to limit the excessive cost of a widespread prescription of expensive targeted drugs. The knowledge of many biomarkers would lead to a personalised cancer treatment. In the meantime, the research on the mechanisms of resistance to moAbs would help to save resources for useless treatment and to discover new drugs to be delivered when the standard ones are no longer effective. The realisation of these goals would yield a radical change in the daily practice for most oncologists. For this reason, this update could be a useful insight in what is coming in their activity for next years.

\section{Acknowledgment}

G Bronte and G Sortino contributed equally to this work.

\section{Declaration of interest}

The authors have no relevant affiliations or financial involvement with any organisation or entity with a financial interest in or financial conflict with the subject matter or materials discussed in the manuscript. This includes employment, consultancies, honoraria, stock ownership or options, expert testimony, grants or patents received or pending or royalties.

\section{Bibliography}

Papers of special note have been highlighted as either of interest $(\bullet)$ or of considerable interest

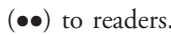

1. Di Fede G, Bronte G, Rizzo S, et al. Monoclonal antibodies and antibody fragments: state of the art and future perspectives in the treatment of nonhaematological tumors. Expert Opin Biol Ther 2011;11(11):1433-45

2. Bronte G, Rizzo S, La Paglia L, et al. Driver mutations and differential sensitivity to targeted therapies: a new approach to the treatment of lung adenocarcinoma. Cancer Treat Rev 2010;36(Suppl 3):S21-9

3. Rolfo C, Giovannetti E, Hong DS, et al. Novel therapeutic strategies for patients with NSCLC that do not respond to treatment with EGFR inhibitors. Cancer Treat Rev 2014;40(8):990-1004

4. Bronte G, Cicero G, Sortino G, et al. Immunotherapy for recurrent ovarian cancer: a further piece of the puzzle or a striking strategy? Expert Opin Biol Ther 2014;14(1):103-14

5. Bronte G, Cicero G, Cusenza S, et al. Monoclonal antibodies in gastrointestinal cancers. Expert Opin Biol Ther 2013;13(6):889-900

6. Rolfo C, Bronte G, Sortino G, et al. The role of targeted therapy for gastrointestinal tumors. Expert Rev Gastroenterol Hepatol 2014;1-11

7. Mitri Z, Constantine T, O'Regan R. The HER2 receptor in breast cancer: pathophysiology, clinical use, and new advances in therapy.

Chemother Res Pract 2012;2012:743193

8. Hudis CA. Trastuzumab-mechanism of action and use in clinical practice. Eng J Med 2007;357(1):39-51

9. Slamon DJ, Leyland-Jones B, Shak S, et al. Use of chemotherapy plus a monoclonal antibody against HER2 for metastatic breast cancer that overexpresses HER2. Eng J Med 2001;344(11):783-92

10. Marty M, Cognetti F, Maraninchi D, et al. Randomized phase II trial of the efficacy and safety of trastuzumab combined with docetaxel in patients with human epidermal growth factor receptor 2-positive metastatic breast cancer administered as first-line treatment: the M77001 study group. J Clin Oncol 2005;23(19):4265-74

11. Gasparini G, Gion M, Mariani L, et al. Randomized Phase II Trial of weekly paclitaxel alone versus trastuzumab plus weekly paclitaxel as first-line therapy of patients with Her- 2 positive advanced breast cancer. Breast Cancer Res Treat 2007;101(3):355-65

12. Pegram MD, Lipton A, Hayes DF, et al. Phase II study of receptor-enhanced chemosensitivity using recombinant humanized anti-p185HER2/neu monoclonal antibody plus cisplatin in patients with HER2/neu-overexpressing metastatic breast cancer refractory to chemotherapy treatment. J Clin Oncol 1998;16(8):2659-71

13. Pegram MD, Pienkowski T, Northfelt DW, et al. Results of two open-label, multicenter phase II studies of docetaxel, platinum salts, and trastuzumab in HER2-positive advanced breast cancer. J Natl Cancer Inst 2004;96(10):759-69

14. Burstein HJ, Keshaviah A, Baron AD, et al. Trastuzumab plus vinorelbine or taxane chemotherapy for HER2overexpressing metastatic breast cancer: the trastuzumab and vinorelbine or taxane study. Cancer 2007;110(5):965-72

15. Yamamoto D, Iwase S, Kitamura K, et al. A phase II study of trastuzumab and capecitabine for patients with HER2-overexpressing metastatic breast cancer: Japan Breast Cancer Research Network (JBCRN) 00 Trial. Cancer Chemother Pharmacol 2008;61(3):509-14

16. Yardley DA, Burris HA, Simons L, et al. A phase II trial of gemcitabine/ carboplatin with or without trastuzumab in the first-line treatment of patients with metastatic breast cancer. Clin Breast Cancer 2008;8(5):425-31

17. Vogel CL, Cobleigh MA, Tripathy D, et al. Efficacy and safety of trastuzumab as a single agent in first-line treatment of HER2-overexpressing metastatic breast cancer. J Clin Oncol 2002;20(3):719-26 


\section{G. Bronte et al.}

18. Nahta R, Yu D, Hung MC, et al. Mechanisms of disease: understanding resistance to HER2-targeted therapy in human breast cancer. Nat Clin Pract Oncol 2006;3(5):269-80

19. Montemurro F, Redana S, Viale G, et al. Retrospective evaluation of clinical outcomes in patients with HER2-positive advanced breast cancer progressing on trastuzumab-based therapy in the prelapatinib era. Clin Breast Cancer 2008;8(5):436-42

20. Pirvulescu C, Uhlig M, von Minckwitz G, GROUP GGB. Trastuzumab improves the efficacy of chemotherapy in breast cancer treatment beyond progression. breast care (Basel) 2008;3(5):364-5

21. Yin W, Jiang $Y$, Shen $Z$, et al. Trastuzumab in the adjuvant treatment of HER2-positive early breast cancer patients: a meta-analysis of published randomized controlled trials. PLoS One 2011;6(6):e21030

22. Goldhirsch A, Gelber RD, Piccart-Gebhart MJ, et al. 2 years versus 1 year of adjuvant trastuzumab for HER2-positive breast cancer (HERA): an open-label, randomised controlled trial. Lancet 2013;382(9897):1021-8

23. Slamon D, Eiermann W, Robert N, et al. Adjuvant trastuzumab in HER2positive breast cancer. Eng J Med 2011;365(14):1273-83

24. Gonzalez-Angulo AM, Litton JK, et al. High risk of recurrence for patients with breast cancer who have human epidermal growth factor receptor 2-positive, nodenegative tumors $1 \mathrm{~cm}$ or smaller. J Clin Oncol 2009;27(34):5700-6

25. Curigliano G, Viale G, Bagnardi V, et al. Clinical relevance of HER2 overexpression/amplification in patients with small tumor size and nodenegative breast cancer. J Clin Oncol 2009;27(34):5693-9

26. McArthur HL, Mahoney KM, Morris PG, et al. Adjuvant trastuzumab with chemotherapy is effective in women with small, node-negative, HER2-positive breast cancer. Cancer 2011;117(24):5461-8

27. Rodrigues MJ, Wassermann J, Albiges L, et al. Trastuzumab treatment in $\mathrm{tlab}$, node-negative, human epidermal growth factor receptor 2-overexpressing breast carcinomas. J Clin Oncol 2010;28(28): $541-2$
28. Olszewski AJ, Migdady Y, Boolbol SK, et al. Effects of adjuvant chemotherapy in HER2-positive or triple-negative pT1ab breast cancers: a multiinstitutional retrospective study. Breast Cancer Res Treat 2013;138(1):215-23

29. Romond EH, Perez EA, Bryant J, et al. Trastuzumab plus adjuvant chemotherapy for operable HER2-positive breast cancer. Eng J Med 2005;353(16):1673-84

30. Perez EA, Suman VJ, Davidson NE, et al. Sequential versus concurrent trastuzumab in adjuvant chemotherapy for breast cancer. J Clin Oncol 2011;29(34):4491-7

31. Wang S, Zheng G, Chen L, Xiong B. Effect of HER-2/neu over-expression on prognosis in gastric cancer: a meta-analysis. Asian Pac J Cancer Prev 2011;12(6):1417-23

32. Bang YJ, Van Cutsem E, Feyereislova A, et al. Trastuzumab in combination with chemotherapy versus chemotherapy alone for treatment of HER2-positive advanced gastric or gastro-oesophageal junction cancer (ToGA): a phase 3, open-label, randomised controlled trial. Lancet 2010;376(9742):687-97

33. Guarneri V, Lenihan DJ, Valero V, et al. Long-term cardiac tolerability of trastuzumab in metastatic breast cancer: the M.D. Anderson Cancer Center experience. J Clin Oncol 2006;24(25):4107-15

34. Rayson D, Richel D, Chia S, et al. Anthracycline-trastuzumab regimens for HER2/neu-overexpressing breast cancer: current experience and future strategies. Ann Oncol 2008;19(9):1530-9

35. Lewis Phillips GD, Li G, Dugger DL, et al. Targeting HER2-positive breast cancer with trastuzumab-DM1, an antibody-cytotoxic drug conjugate. Cancer Res 2008;68(22):9280-90

36. Krop IE, Beeram M, Modi S, et al. Phase I study of trastuzumab-DM1, an HER2 antibody-drug conjugate, given every 3 weeks to patients with HER2positive metastatic breast cancer. J Clin Oncol 2010;28(16):2698-704

37. Junttila TT, Li G, Parsons K, et al. Trastuzumab-DM1 (T-DM1) retains all the mechanisms of action of trastuzumab and efficiently inhibits growth of lapatinib insensitive breast cancer. Breast Cancer Res Treat 2011;128(2):347-56
38. Krop IE, LoRusso P, Miller KD, et al. A phase II study of trastuzumab emtansine in patients with human epidermal growth factor receptor 2positive metastatic breast cancer who were previously treated with trastuzumab, lapatinib, an anthracycline, a taxane, and capecitabine. J Clin Oncol 2012;30(26):3234-41

39. Hurvitz SA, Dirix L, Kocsis J, et al. Phase II randomized study of trastuzumab emtansine versus trastuzumab plus docetaxel in patients with human epidermal growth factor receptor 2-positive metastatic breast cancer. J Clin Oncol 2013;31(9):1157-63

40. Miller KD, Diéras V, Harbeck N, et al. Phase IIa trial of trastuzumab emtansine with pertuzumab for patients with human epidermal growth factor receptor 2-positive, locally advanced, or metastatic breast cancer. J Clin Oncol 2014;32(14):1437-44

41. Verma S, Miles D, Gianni L, et al. Trastuzumab emtansine for HER2positive advanced breast cancer. Eng J Med 2012;367(19):1783-91

- This study led to the approval of trastuzumab emtansine for treatment of HER2-positive, advanced, metastatic breast cancer.

42. Ellis PA BC, Im Y, Patre M, et al. MARIANNE: a Phase III, randomized study of trastuzumab-DM1 (T-DM1) with or without pertuzumab (P) compared with trastuzumab $(\mathrm{H})$ plus taxane for first-line treatment of HER2positive, progressive, or recurrent locally advanced or metastatic breast cancer (MBC). J Clin Oncol 2011;29(Suppl 15):abstract TPS102

43. Krop IE, Kim SB, Gonzalez-Martin A, et al. Trastuzumab emtansine versus treatment of physician's choice for pretreated HER2-positive advanced breast cancer (TH3RESA): a randomised, open-label, phase 3 trial. Lancet Oncol 2014;15(7):689-99

44. Franklin MC, Carey KD, Vajdos FF, et al. Insights into ErbB signaling from the structure of the ErbB2-pertuzumab complex. Cancer Cell 2004;5(4):317-28

45. Scheuer W, Friess T, Burtscher H, et al. Strongly enhanced antitumor activity of trastuzumab and pertuzumab combination treatment on HER2-positive human xenograft tumor models. Cancer Res 2009;69(24):9330-6 
46. Nahta R, Hung MC, Esteva FJ. The HER-2-targeting antibodies trastuzumab and pertuzumab synergistically inhibit the survival of breast cancer cells. Cancer Res 2004;64(7):2343-6

47. Baselga J, Swain SM. CLEOPATRA: a phase III evaluation of pertuzumab and trastuzumab for HER2-positive metastatic breast cancer. Clin Breast Cancer 2010;10(6):489-91

48. Baselga J, Cortés J, Kim SB, et al. Pertuzumab plus trastuzumab plus docetaxel for metastatic breast cancer. Eng J Med 2012;366(2):109-19

49. Swain SM, Kim SB, Cortés J, et al. Pertuzumab, trastuzumab, and docetaxel for HER2-positive metastatic breast cancer (CLEOPATRA study): overall survival results from a randomised, double-blind, placebo-controlled, phase 3 study. Lancet Oncol 2013;14(6):461-71

- This study led to the approval of pertuzumab for treatment of HER2positive, advanced, metastatic breast cancer.

50. Gianni L, Pienkowski T, Im YH, et al. Efficacy and safety of neoadjuvant pertuzumab and trastuzumab in women with locally advanced, inflammatory, or early HER2-positive breast cancer (NeoSphere): a randomised multicentre, open-label, phase 2 trial. Lancet Oncol 2012;13(1):25-32

51. Schneeweiss A, Chia S, Hickish T, et al. Pertuzumab plus trastuzumab in combination with standard neoadjuvant anthracycline-containing and anthracycline-free chemotherapy regimens in patients with HER2-positive early breast cancer: a randomized phase II cardiac safety study (TRYPHAENA). Annals Oncol 2013;24(9):2278-84

52. Jean GW, Shah SR. Epidermal growth factor receptor monoclonal antibodies for the treatment of metastatic colorectal cancer. Pharmacotherapy 2008;28(6):742-54

53. Bronte G, Terrasi M, Rizzo S, et al. EGFR genomic alterations in cancer: prognostic and predictive values. Front Biosci (Elite Ed) 2011;3:879-87

54. Cunningham D, Humblet Y, Siena S, et al. Cetuximab monotherapy and cetuximab plus irinotecan in irinotecanrefractory metastatic colorectal cancer. Eng J Med 2004;351(4):337-45
55. Sobrero AF, Maurel J, Fehrenbacher L, et al. EPIC: phase III trial of cetuximab plus irinotecan after fluoropyrimidine and oxaliplatin failure in patients with metastatic colorectal cancer. J Clin Oncol 2008;26(14):2311-19

56. Van Cutsem E, Köhne CH, Láng I, et al. Cetuximab plus irinotecan, fluorouracil, and leucovorin as first-line treatment for metastatic colorectal cancer: updated analysis of overall survival according to tumor KRAS and BRAF mutation status. J Clin Oncol 2011;29(15):2011-19

57. Russo A, Rizzo S, Bronte G, et al. The long and winding road to useful predictive factors for anti-EGFR therapy in metastatic colorectal carcinoma: the KRAS/BRAF pathway. Oncology 2009;77(Suppl 1):57-68

58. Rizzo S, Bronte G, Fanale D, et al. Prognostic vs predictive molecular biomarkers in colorectal cancer: is KRAS and BRAF wild type status required for anti-EGFR therapy? Cancer Treat Rev 2010;36(Suppl 3):S56-61

59. Tabernero J, Van Cutsem E, Díaz-Rubio E, et al. Phase II trial of cetuximab in combination with fluorouracil, leucovorin, and oxaliplatin in the first-line treatment of metastatic colorectal cancer. J Clin Oncol 2007;25(33):5225-32

60. Maughan TS, Adams RA, Smith CG, et al. Addition of cetuximab to oxaliplatin-based first-line combination chemotherapy for treatment of advanced colorectal cancer: results of the randomised phase 3 MRC COIN trial. Lancet 2011;377(9783):2103-14

61. Tveit KM, Guren T, Glimelius B, et al. Phase III trial of cetuximab with continuous or intermittent fluorouracil, leucovorin, and oxaliplatin (Nordic FLOX) versus FLOX alone in first-line treatment of metastatic colorectal cancer: the NORDIC-VII study. J Clin Oncol 2012;30(15):1755-62

62. Santini D, Vincenzi B, Addeo R, et al. Cetuximab rechallenge in metastatic colorectal cancer patients: how to come away from acquired resistance? Ann Oncol 2012;23(9):2313-18

63. Bonner JA, Harari PM, Giralt J, et al. Radiotherapy plus cetuximab for locoregionally advanced head and neck cancer: 5-year survival data from a phase 3 randomised trial, and relation between cetuximab-induced rash and survival. Lancet Oncol 2010;11(1):21-8

64. Vermorken JB, Mesia R, Rivera F, et al. Platinum-based chemotherapy plus cetuximab in head and neck cancer. Eng J Med 2008;359(11):1116-27

65. Giusti RM, Shastri K, Pilaro AM, et al. U.S. Food and Drug Administration approval: panitumumab for epidermal growth factor receptor-expressing metastatic colorectal carcinoma with progression following fluoropyrimidine-, oxaliplatin-, and irinotecan-containing chemotherapy regimens. Clin Cancer Res 2008;14(5):1296-302

66. Amado RG, Wolf M, Peeters M, et al. Wild-type KRAS is required for panitumumab efficacy in patients with metastatic colorectal cancer. J Clin Oncol 2008;26(10):1626-34

67. Douillard JY, Siena S, Cassidy J, et al. Final results from PRIME: randomized phase 3 study of panitumumab with FOLFOX4 for first-line treatment of metastatic colorectal cancer. Annals Oncol 2014;25(7):1346-55

68. Douillard JY, Oliner KS, Siena S, et al. Panitumumab-FOLFOX4 treatment and RAS mutations in colorectal cancer. Eng J Med 2013;369(11):1023-34

- $\quad$ This study led to the discovery of RAS mutations as predictive biomarker for anti-EGFR moAbs efficacy.

69. Peeters M, Price TJ, Cervantes A, et al. Final results from a randomized phase 3 study of FOLFIRI $\{+/-\}$ panitumumab for second-line treatment of metastatic colorectal cancer. Annals Oncol 2014;25(1):107-16

70. Stintzing S, Fischer von Weikersthal L, Decker $\mathrm{T}$, et al. FOLFIRI plus cetuximab versus FOLFIRI plus bevacizumab as first-line treatment for patients with metastatic colorectal cancersubgroup analysis of patients with KRAS: mutated tumours in the randomised German AIO study KRK-0306. Annals Oncol 2012;23(7):1693-9

71. Fortunato Ciardiello H-JL, et al. Treatment outcome according to tumor RAS mutation status in CRYSTAL study patients with metastatic colorectal cancer (mCRC) randomized to FOLFIRI with/ without cetuximab. J Clin Oncol 2014;32(Suppl 5s):abstract 3506

72. Kaelin WG. The von Hippel-Lindau protein, HIF hydroxylation, and oxygen 
sensing. Biochem Biophys Res Commun 2005;338(1):627-38

73. Folkman J. Tumor angiogenesis: therapeutic implications. Eng J Med 1971;285(21):1182-6

74. Ferrara N, Gerber HP, LeCouter J. The biology of VEGF and its receptors. Nat Med 2003;9(6):669-76

75. Perrot-Applanat M. VEGF isoforms. Cell Adh Migr 2012;6(6):526-7

76. Ferrara N, Hillan KJ, Gerber HP, Novotny W. Discovery and development of bevacizumab, an anti-VEGF antibody for treating cancer. Nat Rev Drug Discov 2004;3(5):391-400

77. Jain RK. Normalization of tumor vasculature: an emerging concept in antiangiogenic therapy. Science 2005;307(5706):58-62

78. Kabbinavar FF, Schulz J, McCleod M, et al. Addition of bevacizumab to bolus fluorouracil and leucovorin in first-line metastatic colorectal cancer: results of a randomized phase II trial. J Clin Oncol 2005;23(16):3697-705

79. Hurwitz H, Fehrenbacher L, Novotny W, et al. Bevacizumab plus irinotecan, fluorouracil, and leucovorin for metastatic colorectal cancer. Eng J Med 2004;350(23):2335-42

80. Saltz LB, Clarke S, Díaz-Rubio E, et al. Bevacizumab in combination with oxaliplatin-based chemotherapy as firstline therapy in metastatic colorectal cancer: a randomized phase III study. J Clin Oncol 2008;26(12):2013-19

81. Caraglia M, Santini D, Bronte G, et al. Predicting efficacy and toxicity in the era of targeted therapy: focus on anti-EGFR and anti-VEGF molecules. Curr Drug Metab 2011;12(10):944-55

82. Rolfo C, Russo A, Santini D, et al. Dilemma in metastatic colorectal cancer: VEGF versus EGRF targeting. Expert Opin Ther Targets 2013;17(8):869-71

83. Bronte G, Rolfo C, Peeters M, Russo A. How to find the Ariadne's thread in the labyrinth of salvage treatment options for metastatic colorectal cancer? Expert Opin Biol Ther 2014;14(6):743-8

84. Bennouna J, Sastre J, Arnold D, et al. Continuation of bevacizumab after first progression in metastatic colorectal cancer (ML18147): a randomised phase 3 trial. Lancet Oncol 2013;14(1):29-37
85. Kubicka S, Greil R, André T, et al. Bevacizumab plus chemotherapy continued beyond first progression in patients with metastatic colorectal cancer previously treated with bevacizumab plus chemotherapy: ML18147 study KRAS subgroup findings. Annals Oncol 2013;24(9):2342-9

86. Fotios Loupakis CC, Lonardi S, et al. Subgroup analyses in RAS mutant, BRAF mutant and all-wt mCRC pts treated with FOLFOXIRI plus bevacizumab (bev) or FOLFIRI plus bev in the TRIBE study. J Clin Oncol 2014;32(Suppl 5):abstract 3519

87. Sandler A, Gray R, Perry MC, et al. Paclitaxel-carboplatin alone or with bevacizumab for non-small-cell lung cancer. Eng J Med 2006;355(24):2542-50

88. Johnson DH, Fehrenbacher L, Novotny WF, et al. Randomized phase II trial comparing bevacizumab plus carboplatin and paclitaxel with carboplatin and paclitaxel alone in previously untreated locally advanced or metastatic non-small-cell lung cancer. J Clin Oncol 2004;22(11):2184-91

89. Reck M, von Pawel J, Zatloukal P, et al. Phase III trial of cisplatin plus gemcitabine with either placebo or bevacizumab as first-line therapy for nonsquamous non-small-cell lung cancer: AVAil. J Clin Oncol 2009;27(8):1227-34

90. Miller K, Wang M, Gralow J, et al. Paclitaxel plus bevacizumab versus paclitaxel alone for metastatic breast cancer. Eng J Med 2007;357(26):2666-76

91. Miles DW, Chan A, Dirix LY, et al. Phase III study of bevacizumab plus docetaxel compared with placebo plus docetaxel for the first-line treatment of human epidermal growth factor receptor 2-negative metastatic breast cancer. J Clin Oncol 2010;28(20):3239-47

92. Robert NJ, Diéras V, Glaspy J, et al. RIBBON-1: randomized, double-blind, placebo-controlled, phase III trial of chemotherapy with or without bevacizumab for first-line treatment of human epidermal growth factor receptor 2-negative, locally recurrent or metastatic breast cancer. J Clin Oncol 2011;29(10):1252-60

93. Escudier B, Pluzanska A, Koralewski P, et al. Bevacizumab plus interferon alfa- 2 a for treatment of metastatic renal cell carcinoma: a randomised, double-blind phase III trial. Lancet 2007;370(9605):2103-11

94. Escudier B, Bellmunt J, Négrier $S$, et al. Phase III trial of bevacizumab plus interferon alfa-2a in patients with metastatic renal cell carcinoma (AVOREN): final analysis of overall survival. J Clin Oncol 2010;28(13):2144-50

95. Rini BI, Halabi S, Rosenberg JE, et al. Phase III trial of bevacizumab plus interferon alfa versus interferon alfa monotherapy in patients with metastatic renal cell carcinoma: final results of CALGB 90206. J Clin Oncol 2010;28(13):2137-43

96. Friedman HS, Prados MD, Wen PY, et al. Bevacizumab alone and in combination with irinotecan in recurrent glioblastoma. J Clin Oncol 2009;27(28):4733-40

97. Kreisl TN, Kim L, Moore K, et al. Phase II trial of single-agent bevacizumab followed by bevacizumab plus irinotecan at tumor progression in recurrent glioblastoma. J Clin Oncol 2009;27(5):740-5

98. Burger RA, Brady MF, Bookman MA, et al. Incorporation of bevacizumab in the primary treatment of ovarian cancer. Eng J Med 2011;365(26):2473-83

99. Oza AM, Perren TJ, Swart AM, et al. LATE BREAKING ABSTRACT: ICON7: Final overall survival results in the GCIG phase III randomized trial of bevacizumab in women with newly diagnosed ovarian cancer [abstract 6]. European Cancer Congress; 29 September 2013

100. Fuchs CS, Tomasek J, Yong CJ, et al. Ramucirumab monotherapy for previously treated advanced gastric or gastro-oesophageal junction adenocarcinoma (REGARD): an international, randomised, multicentre, placebo-controlled, phase 3 trial. Lancet 2014;383(9911):31-9

- This study led to the approval of ramucirumab for the treatment of patients with advanced or metastatic, gastric or gastroesophageal junction cancer adenocarcinoma.

101. Wilke H, Van Cutsem E, Cheul Oh S, et al. RAINBOW: a global, phase III, randomized, double-blind study of ramucirumab plus paclitaxel versus 
placebo plus paclitaxel in the treatment of metastatic gastroesophageal junction (GEJ) and gastric adenocarcinoma following disease progression on first-line platinum- and fluoropyrimidinecontaining combination therapy rainbow IMCL CP12-0922 (I4T-IE-JVBE).

$\mathrm{J}$ Clin Oncol

2014;32(Suppl 3):abstract LBA7

102. Van Cutsem E, Tabernero J, Lakomy R, et al. Addition of aflibercept to fluorouracil, leucovorin, and irinotecan improves survival in a phase III randomized trial in patients with metastatic colorectal cancer previously treated with an oxaliplatin-based regimen. J Clin Oncol 2012;30(28):3499-506

- This study led to the approval of aflibercept for treatment of metastatic colorectal cancer patients.

103. Maker AV, Attia P, Rosenberg SA. Analysis of the cellular mechanism of antitumor responses and autoimmunity in patients treated with CTLA- 4 blockade. J Immunol (Baltimore, MD : 1950) 2005;175(11):7746-54

104. Hodi FS, O’Day SJ, McDermott DF, et al. Improved survival with ipilimumab in patients with metastatic melanoma. Eng J Med 2010;363(8):711-23

105. Robert C, Thomas L, Bondarenko I, et al. Ipilimumab plus dacarbazine for previously untreated metastatic melanoma. Eng J Med 2011;364(26):2517-26

- This study led to the approval of ipilimumab for treatment of patients with metastatic melanoma.

106. Lynch TJ, Bondarenko I, Luft A, et al. Ipilimumab in combination with paclitaxel and carboplatin as first-line treatment in stage IIIB/IV non-small-cell lung cancer: results from a randomized, double-blind, multicenter phase II study. J Clin Oncol 2012;30(17):2046-54

107. Reck M, Bondarenko I, Luft A, et al. Ipilimumab in combination with paclitaxel and carboplatin as first-line therapy in extensive-disease-small-cell lung cancer: results from a randomized, double-blind, multicenter phase 2 trial. Ann Oncol 2013;24(1):75-83

108. Reck M, Lu H, Gribkoff G, et al. CA184-104: Randomized, multicenter, double-blind, phase III trial comparing the efficacy of ipilimumab (Ipi) with paclitaxel/carboplatin (PC) versus placebo with PC in patients (pts) with stage IV/ recurrent non-small cell lung cancer (NSCLC) of squamous histology. $\mathrm{J}$ Clin Oncol 2012;30(Suppl):abstract TPS7611

109. Von Pawel J, Kim SW, Spigel DR, et al. CA184-156: Randomized, multicenter, double-blind, phase III trial comparing the efficacy of ipilimumab (Ipi) plus etoposide/platinum (EP) versus placebo plus EP in patients (Pts) with newly diagnosed extensive-stage disease small cell lung cancer (ED-SCLC).

$\mathrm{J}$ Clin Oncol 2013;31(Suppl):abstract TPS7608

110. Weilbaecher KN, Guise TA, McCauley LK. Cancer to bone: a fatal attraction. Nat Rev Cancer 2011;11(6):411-25

111. Lipton A, Fizazi K, Stopeck AT, et al. Superiority of denosumab to zoledronic acid for prevention of skeletal-related events: a combined analysis of 3 pivotal, randomised, phase 3 trials. Eur J Cancer 2012;48(16):3082-92

-• This study led to the approval of denosumab for prevention of skeletalrelated events in patients with bone metastases from solid tumours.

112. Smith MR, Saad F, Coleman R, et al. Denosumab and bone-metastasis-free survival in men with castration-resistant prostate cancer: results of a phase 3 , randomised, placebo-controlled trial. Lancet 2012;379(9810):39-46

113. Thatcher N, Hirsch FR, Szczesna A, et al. A randomized, multicenter, openlabel, phase III study of gemcitabinecisplatin (GC) chemotherapy plus necitumumab (IMC-11F8/LY3012211) versus GC alone in the first-line treatment of patients (pts) with stage IV squamous non-small cell lung cancer (sqNSCLC). J Clin Oncol 2014;32(Suppl 5s):abstract 8008^

114. Luis Paz-Ares JM, Mezger J, Ciuleanu T, et al. Randomized phase- 3 trial (INSPIRE) of necitumumab plus cisplatin-pemetrexed versus cisplatinpemetrexed alone as first-line therapy in stage IV non-squamous NSCLC.
J Thorac Oncol 2013;8(Suppl 2):S1-S1410

115. Ribas A, Kefford R, Marshall MA, et al. Phase III randomized clinical trial comparing tremelimumab with standard-of-care chemotherapy in patients with advanced melanoma. J Clin Oncol 2013;31(5):616-22

116. Topalian SL, Hodi FS, Brahmer JR, et al. Safety, activity, and immune correlates of anti-PD-1 antibody in cancer. Eng J Med 2012;366(26):2443-54

117. Herbst RS, Gordon MS, Fine GD, et al. A study of MPDL3280A, an engineered PD-L1 antibody in patients with locally advanced or metastatic tumors. J Clin Oncol 2013;31(Suppl):abstract 3000

118. Spigel DR, Ervin TJ, Ramlau RA, et al. Randomized phase II trial of Onartuzumab in combination with erlotinib in patients with advanced non-small-cell lung cancer. J Clin Oncol 2013;31(32):4105-14

119. Spigel DR. Onartuzumab plus erlotinib versus erlotinib in previously treated stage IIIb or IV NSCLC: Results from the pivotal phase III randomized, multicenter, placebo-controlled METLung (OAM4971g) global trial. J Clin Oncol 2014;32(Suppl 5s):abstract 8000

\section{Affiliation}

Giuseppe Bronte ${ }^{1}$, Giovanni Sortino ${ }^{1}$, Francesco Passiglia ${ }^{1}$, Sergio Rizzo ${ }^{1}$, Francesca Lo Vullo ${ }^{1}$, Antonio Galvano ${ }^{1}$, Viviana Bazan ${ }^{1}$, Christian Rolfo ${ }^{2} \&$ Antonio Russo ${ }^{\dagger 3} \mathrm{MD} \mathrm{PhD}$

${ }^{\dagger}$ Author for correspondence

${ }^{1}$ University of Palermo, Department of Surgical, Oncological and Oral Sciences, Palermo, Italy

${ }^{2}$ Antwerp University Hospital, Oncology Department, Phase I - Early Clinical Trials Unit, Edegem, Belgium

${ }^{3}$ Professor,

University of Palermo, Department of Surgical, Oncological and Oral Sciences, Section of Medical Oncology, Via del Vespro 129 - 90127 Palermo, Italy

Tel: +39091 6552500;

Fax: +39 0916554529 ;

E-mail: antonio.russo@usa.net 